\title{
Role of Lipoproteins in \\ Carcinogenesis and in Chemoprevention
}

\author{
Adebowale Bernard Saba and Temitayo Ajibade
}

Additional information is available at the end of the chapter

http://dx.doi.org/10.5772/46065

\section{Introduction}

Lipoproteins are complex aggregates of lipids and proteins that render endogenous lipids compatible with the aqueous environment of body fluids (Brown, 2007). The major physiological role of lipoproteins is to transport water-insoluble lipids from their point of origin to their respective destinations. Lipoproteins are synthesised mainly in the liver and intestines. They are in a state of constant flux, in circulation, changing in composition and physical structure as the peripheral tissues take up the various components before the remnants are returned to the liver. The most abundant lipid constituents of lipoproteins are free cholesterol, cholesterol esters, triacylglycerols and phospholipids, though fat-soluble vitamins and anti-oxidants are also found in lipoproteins (Kwiterovich, 2000).

\section{Classification of lipoproteins}

Lipoproteins are classified as chylomicrons (CM), very-low-density lipoproteins (VLDL), low-density lipoproteins (LDL) and high-density lipoproteins (HDL), based on the relative densities of the aggregates on ultracentrifugation. These classes are further refined by improved separation procedures, and intermediate-density lipoproteins (IDL) and subdivisions of the HDL (e.g. HDL1, HDL2, HDL3 etc) are often defined. Density of lipoproteins is determined largely by the relative concentrations of triacylglycerols and proteins and by the diameters of the broadly spherical particles, which vary from about $6000 \AA$ in $C M$ to $100 \AA$ or less in the smallest HDL. An alternative nomenclature is based on the relative mobilities on electrophoresis on agarose gels. Thus, $\alpha$, pre- $\beta$ and $\beta$ lipoproteins correspond to HDL, VLDL and LDL, respectively (Lacko et al., 2007).

\section{Chylomicrons}

Chylomicrons, the largest and least dense of the lipoproteins are formed in the intestinal cell walls from dietary fat and cholesterol. Their main task is to carry triglycerides from the 
intestine to the tissues where they are needed as a source of energy. In the circulation, triglycerides are removed from chylomicrons via the action of lipoprotein lipase (LPL), an enzyme present in the capillaries of many tissues. If present in large amounts, such as after a fatty meal, chylomicrons cause the plasma to appear milky.

\section{Very low density lipoproteins}

Very low density lipoproteins (VLDLs) are synthesised in the liver. Much like chylomicrons, they function primarily to distribute triglycerides to target sites such as adipose tissue and skeletal muscle where they are used for storage and energy. The manner in which triglycerides are removed from the circulation is the same as that of chylomicrons. Gradually with removal of triglycerides and protein, VLDLs are converted to LDL. High plasma levels of VLDL are associated with familial hypertriglyceridaemia, diabetes mellitus and underactive thyroid.

\section{Low density lipoproteins}

Low density lipoproteins are cholesterol-rich particles. About 70\% of plasma cholesterol occurs in this form. LDLs are chiefly involved in the transport of the cholesterol manufactured in the liver to the tissues, where it is used. Uptake of cholesterol into cells occurs when lipoprotein binds to LDL receptors on the cell surface. LDL is then taken into the cell and broken down into free cholesterol and amino acids. Disorders involving a defect in or lack of LDL receptors are usually characterised by high plasma cholesterol levels. The cholesterol cannot be cleared efficiently from the blood and therefore accumulates.

\section{High density lipoproteins}

The high-density lipoproteins (HDLs) are small, dense, and spherical lipid-protein complexes which are normally considered to consist of those plasma lipoprotein particles which fall into the density range of $1.063-1.210 \mathrm{~g} / \mathrm{mL}$. HDL particles are composed of an outer layer containing free cholesterol, phospholipid, and various apolipoproteins (Apo), which covers a hydrophobic core consisting primarily of triglycerides and cholesterol esters (Barter et al., 2003). The major proteins are Apo A-I (Mr 28,000) and Apo A-II (Mr 17,000). Apo A-I, the primary protein constituent of these particles, accounts for about $60 \%$ of the protein content of HDL. Apo A-I is synthesized in the intestines and liver and is thought to be largely responsible for the antiatherogenic effects of HDL. Some HDL particles carry only Apo A-I, whereas others contain both Apo A-I and Apo A-II (Shah et al., 2001). Other apolipoprotein species found in HDL particles include Apo A-IV, Apo C (C-I, C-II, and C-III), and Apo E.

\section{High density lipoprotein subtypes}

Several subtypes of HDL particles have been identified on the basis of density, electrophoretic mobility, particle size, and apolipoprotein composition (Albers et al., 1984). Differences in particle size are mainly the result of the number of apolipoprotein particles and the volume of the cholesterol ester in the core of the particle. HDL can also be classified into larger, less dense HDL2 or smaller, denser HDL3 which falls within the density ranges $1.063-1.125$ and $1.125-1.210 \mathrm{~g} / \mathrm{mL}$, respectively. Although the major proportion of HDL is 
normally present in HDL3, individual variability in HDL levels in human populations usually reflects different amounts of HDL2 (Skinner, 1994). HDL2 is richer in particles containing Apo A-I without Apo A-II, whereas HDL3 is richer in particles containing both Apo A-I and Apo A-II (Gotto, 2001).

\section{Synthesis, Metabolism and Regulation of plasma lipoprotein concentration}

Normal metabolism and homeostasis of carbohydrates, amino acids and lipids in vivo depend on integrated liver function. Most plasma apolipoproteins and endogenous lipids and lipoproteins, including apolipoprotein(a) (apo(a)) and lipoprotein(a) (Lp(a)), are synthesized in the liver. The apolipoprotein(a) is a high molecular weight glycoprotein, of 250-838 kD (Bowden, 1994) and a total of 34 different apo(a) isoforms have been identified in populations (Marcovina et al., 1993). The core components of Lp(a) are neutral lipid and an apoB-100 molecule, which are covalently connected by a disulfide-bond bridge and surrounded by hydrophilic apo(a) (Byrne, 1994). The heterogeneity of apo(a) determines the changes in plasma $\mathrm{Lp}(\mathrm{a})$ concentrations, and there is a negative correlation between the molecular weight of apo(a) and the plasma Lp(a) concentration (Wade, 1993)

Lp(a) has a simple Mendelian dominant inheritance, which is controlled by the alleles Lpa and Lp0 (Utermann et al., 1988). Plasma Lp(a) concentration is controlled by three alleles., i.e., LpA, Lpa and Lp0 (Hasstedt et al. 1986). Pedigree analysis indicated that the size polymorphism of $\mathrm{Lp}(\mathrm{a})$ is controlled by a series of alleles of a single point (Utermann, 1988). The apo(a) gene which is located in q26-27 of chromosome 6 in humans has a linkage to the plasminogen (PGN) gene, and is inherited in a codominant Mendelian model (Amemiya, 1996). Apolipoprotein(a) mRNA (14 kb) encodes for a mature protein of 4529 amino acid residues in the presence of a signal peptide with 19 amino acid residues (McLean, 1987) while a high-degree homology exists between the molecular structures of the apo(a) gene and the PGN gene. The high-degree homology between the apo(a) gene and the PGN gene determines the biological actions of Lp(a) (Romics et al., 1996).

\section{Role of lipoprotein in cancer}

Cancerous cells generally have high requirements for cholesterol as they are rapidly dividing cells. The Low density lipoproteins (LDL) which are cholesterol-rich particles have been especially found to play significant role in the pathogenesis of a large number of cancers. For instance, increased LDL requirement and receptor activity have been reported in cancer of the prostate gland (Chen and Hughes-Fulford, 2001); colon (Niendorf et al., 1995); adrenal gland (Nakagawa et al., 1995); hormone unresponsive breast tumors (Stranzl et al., 1997), cancers of gynecological origin, tumors of lung tissues (Vitols et al., 1992), leukemia (Tatidis et al., 2002), and malignant brain tumors (Rudling et al., 1990). In contrast, high density lipoproteins (HDL) have been reported significantly lowered in patients with primary or metastatic liver cancer (Moorman et al., 1998). Hoyer and Engohm (1992) observed an inverse association between serum HDL-cholesterol and risk of breast cancer in a cohort of 5,207 Danish women, who participated in the Glostrup population studies of breast cancer. 
Lymphoma patients often exhibit abnormal lipid metabolism. Numerous clinical studies of lymphoma patients have reported lipid abnormalities that are similar to the dyslipidemia observed in inflammatory and infectious diseases that are believed to develop secondary to circulating cytokines and the accompanying acute-phase response (Blackman et al., 1993). Spiegel et al. (1989) investigated plasma lipids and lipoproteins at presentations in 25 patients with acute leukemia and non-Hodgkin's lymphoma and reported that all patients demonstrated an abnormality in at least one plasma lipid fraction and most exhibited a predictable pattern of lipid alterations that consisted of extremely low levels of HDLcholesterol, elevated triglyceride, and elevated very low density lipoprotein (VLDL). The degree of lipid abnormality was directly related to the underlying tumor burden and particularly to the presence of bone marrow involvement. Therefore, low levels of circulating HDL-cholesterol in lymphoma patients may occur before the clinical onset of cancer and may serve as a marker for inflammation-induced lymphomagenesis, rather than a consequence of lymphoma-induced acute-phase responses.

\subsection{Role of lipoprotein(a) in pathogenesis of cancer}

Lipoprotein[a] is an intriguing molecule consisting of a low-density lipoprotein core and a covalently bound apolipoprotein[a]. Apolipoprotein[a] possess an inactive protease domain which is a single copy of the plasminogen kringle 5 and multiple repeats of domains homologous to the plasminogen kringle 4 . The plasminogen kringle 5 (K5) domain, which is distinct from angiostatin, possesses potent anti-angiogenic properties on its own, which can be exploited in cancer therapy. The angiostatic effect and novel proinflammatory role of the $\mathrm{K} 5$ protein is via its ability to recruit tumor-associated neutrophils and NKT lymphocytes, leading to a potent antitumor response (Perri et al., 2007).

Recently, anti-angiogenic agents have been found to promote leucocyte-vessel wall interaction as part of their anti-tumorigenic effects. Studies on animal models have indicated that the proteolytic break-down products of apolipoprotein[a] may posses anti-angiogenic and anti-tumorigenic effects both in vitro and in vivo. This is a convenient premise to develop novel therapeutic modalities which may efficiently suppress tumor growth and metastasis (Giuseppe et al., 2007). Significant decrease in Lipoprotein[a] levels have been reported in liver cancer patients by Samonakis et al., 2004. Although the liver plays an important role in lipid metabolism, several non-hepatic factors such as hormones, cytokines, genetics and nutrition are also involved in different ways. For example, several inflammatory and tumoral diseases are characterized by the production and delivery of cytokines influencing serum Lipoprotein[a] levels.

The mechanisms by which cancers induce cachexia involve inflammatory cytokine production; which is responsible for a wide number of metabolic disorders, essentially involving lipid metabolism (Langstein and Norton, 1991) and serum Lipoprotein[a] level changes during inflammatory disease. Liver damage has been linked to reduce Lipoprotein[a] serum levels (Malaguarnera et al., 1996). Geiss et al.(1996) observed marked increase in Lipoprotein[a] concentration from $7 \mathrm{mg} / \mathrm{dl}$ in acute stage to $32 \mathrm{mg} / \mathrm{dl}$ in convalescence in hepatitis 
patients.The lipoprotein[a] half-life is short in vivo, 3.3 3.9 d, (Krempler et al., 1983), and influenced early by liver function alterations (Malaguarnera et al., 1994), The high involvement of Lipoprotein[a] in lipid and protein metabolism have been suggested to be a sensitive and early marker of liver malfunction, therefore Lipoprotein[a] may supply useful additional information for a more complete assessment and monitoring of the liver function in patients with hepatocellular carcinoma and liver cirrhosis (Uccello et al., 2011).

\subsubsection{Role of high density lipoprotein (HDL) in the pathogenesis of cancer}

The origin and fate of HDL are less well understood than other lipoproteins. HDL may be formed both in the intestine and in the liver. It is also formed during lipolysis of Triglycerides-rich lipoproteins. Apolipoprotein AI (ApoAI) and apolipoprotein AII (apoAII) are the major apolipoproteins of HDL and the production rate of apoA-I is an important determinant of the variability of plasma HDL concentrations. Production rate of apoA-I is however, influenced by many factors and apoA-I transcriptional regulation has an impact on plasma HDL concentrations.

A large number of cellular lipid transporters and receptors including a spectrum of HDL and intermediates of HDL participate in the transport of excess cholesterol from peripheral cells to the liver. At one end of the spectrum are lipid-free or lipid-poor apoA-I particles, referred to as pre- $\beta$ HDL. These particles are secreted by the liver and small intestine or generated from surface material from partially lipolyzed chylomicrons or from HDL2 in the periphery by the action of cholesteryl ester transfer protein (CETP), hepatic lipase, or phospholipid transfer protein (PTP) (Dullaart et al., 2001). Lipolyzed chylomicrons are HDL precursors that accept unesterified cholesterol and phospholipids transferred by ATP-binding cassette transporter A1 (ABCA1) on peripheral cells, giving rise to discoidal lipoproteins containing apoA-I (Yokoyama, 2005). The acquired unesterified cholesterol of the apoA-I is esterified by the plasma enzyme lecithin:cholesterol acyltransferase (LCAT) to form cholesteryl ester which is packaged into the hydrophobic core of the discoidal particle, converting it to spherical HDL3. HDL3 can continue to accept unesterified cholesterol and phospholipids from the class B, type I scavenger receptor (SR-BI); through continued action of LCAT (Fig. 1). This causes the hydrophobic core of the discoidal lipoprotein particle to expand and the size increases, thereby forming HDL2.

High density lipoprotein (HDL) plays a key role in the reverse cholesterol transport pathway (RCTP) (Genest et al., 1990) and various fractions of HDL have been shown to offer a new approach to study liver diseases (Cooper et al., 1996). Plasma HDL-C, HDL-PL and HDL-C/HDL-PL have been reported to be lower in hepatocellular carcinoma patients than those in normal patients (Li et al., 1993). In a study on 40 patients with hepatocellular carcinoma, LDL-C level was found to be significantly lower in the hepatocellular carcinoma patients than in the controls, but HDL-C did not show a statistically significant difference to the controls (Motta et al., 2001). HDL-C itself has also been reported significantly decreased in patients with primary or metastatic liver cancer (Kanel et al. 1983). Therefore, variations in the level of plasma lipids and lipoproteins may assist in describing the nature of cirrhosis and hepatocellular carcinoma (Ahaneku et al., 1992). 


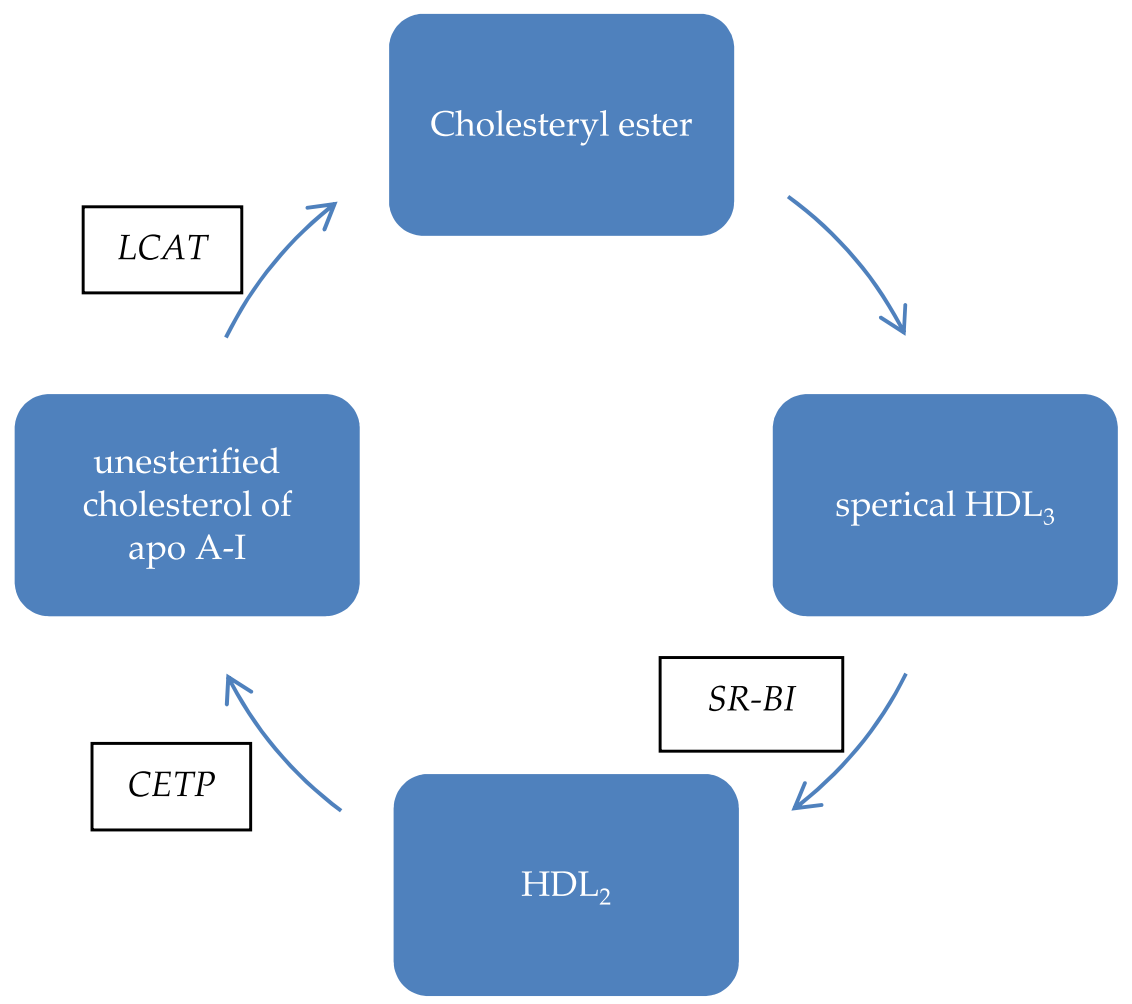

Figure 1. Formation of $\mathrm{HDL}_{2}$ from unesterified cholesterol

Furthermore, an inverse and significant association exists between levels of HDL cholesterol and the risk of incident cancer, according to the results of a recent study (Jafri et al., 2010). Exceptionally, a few cancer risk factors are associated with increased levels of HDL. For instance, many breast cancer risk factors are associated with high HDL-C and the relationship between breast cancer and HDL-C is independent of other risk factors (Moorman et al., 1998).

HDL could play a role in carcinogenesis through its influence on cell cycle entry, via a mitogen-activated protein kinase-dependent pathway or regulation of apoptosis. Specifically, an inverse association exists between serum HDL and risk of breast cancer, and several studies have reported lower levels of HDL in breast cancer patients. Further studies have also shown that tumor progression from localized to metastatic disease is associated with declining HDL levels. At least two population-based screening surveys involving Norwegian women have established low HDL, as part of the metabolic syndrome associated with increased post-menopausal breast cancer risk. The risk of postmenopausal breast cancer among overweight and obese women in the highest serum HDL-cholesterol quartile was one-third the risk of women in the lowest serum HDLcholesterol quartile (Furberg et al., 2004). 


\subsection{Serum lipid profile and incidence of cancer}

Cancer patients often present altered serum lipid profile including changes of HDL level. Case-control studies of newly diagnosed lung cancer have shown that HDL levels are reduced in lung cancer cases. Patients with advanced nonresectable lung cancer also often have decreased serum HDL. Although the biological mechanisms that might link low plasma levels of HDL with cancer are not well understood, the HDL regulation of cell cycle entry through a mitogen activated protein kinase-dependent pathway and apoptosis, modulation of cytokine production, and antioxidative function (Perletti et al., 1996) has been suggested biologically plausible.

The association between higher HDL and lower overall cancer incidence observed in the ATBC cohort is biologically plausible, as HDL has anti-inflammatory properties (Perletti et al., 1996). However, it is also plausible that this association reflects the effect of factors such as inflammation, which are associated with both HDL and risk of cancer.

\subsection{Effect of inflammation on HDL levels in the body}

Inflammation reduces HDL and likely increases risk of lung cancer (Mendez et al., 1991). Studies have shown that chronic inflammation is known to reduce both serum HDLcholesterol levels and its anti-inflammatory properties. The lipoprotein abnormalities seen in patients with inflammatory diseases are thought to develop secondary to circulating cytokines and the accompanying acute-phase response (Blackman et al., 1993). Low HDLcholesterol may therefore be a marker for the severity of systemic inflammation and inflammation-induced non-Hodgkin's lymphoma risk. Conversely, high HDL-cholesterol itself may be protective against non-Hodgkin's lymphoma. High-density lipoproteincholesterol seems to modulate inflammatory responses independent of non-HDL cholesterol levels by suppressing chemotactic activity of monocytes and lymphocytes and inhibiting cytokine-induced expression of endothelial cell adhesion molecules.

\subsection{Association between antioxidant enzymes and HDL}

There are a number of enzymes associated with HDL that have antioxidant properties, including paraoxonase, platelet-activating factor acetylhydrolase, and glutathione peroxidase. Paraoxonase-1 (PON1), the enzyme primarily responsible for HDL's antioxidant function, is closely bound to the HDL particle. PON1's enzymatic activity is highly regulated by environmental factors such as diet and physical activity, by certain drugs, and by genetic factors, especially certain genetic polymorphisms in the paraoxonase- 1 gene, PON1. PON1 is synthesized in the mammalian liver and circulates in blood bound to HDL apolipoprotein (apo) A-1 and apo J. There are 2 other proteins in the same family as PON1 that probably also have antioxidant actions. These are PON2 and PON3. PON2 is ubiquitously expressed within cells, whereas PON3 exhibits a basal constitutive antioxidant activity and is essentially bound to HDL (Reddy et al., 2001; Ng et al., 2001). These enzymes have the ability to prevent the formation of proinflammatory oxidized phospholipids and to block the 
activity of those already formed; however, these oxidized lipids negatively regulate the activities of the HDL-associated enzymes. During an acute-phase response in rabbits, mice, and humans, there seems to be an increase in the formation of these oxidized lipids that results in the inhibition of the HDL-associated enzymes and an association of acute-phase proteins with HDL that renders HDL pro-inflammatory rather than being antiinflammatory.

\subsection{Role of lipoproteins in cancer chemotherapy}

The efficacy of cancer chemotherapy is often limited by severe cytotoxic effects induced by anticancer drugs, on healthy and cancerous cells (Sehouli et al., 2002). In addition, most of the available dosage forms perform with less than optimal efficiency because of poor solubility and limited accessibility to target tissues (Rosen and Abribat, 2005). Furthermore, it is difficult to eradicate cancer cells in vivo because they share the same biochemical machinery with normal cells.

In spite of the overwhelmingly large number of anticancer drugs that have been developed, none is completely selective for cancer cells. Consequently, all anticancer drugs presently in use induce significant dose-limiting toxic side effects. For this reason, there has been increased emphasis on selective delivery of drugs to tumours in ways that bypass normal body tissues.

\subsubsection{Lipoproteins as special anticancer drug delivery agent}

The cytotoxic effects of cancer chemotherapeutic drugs on healthy organs can be significantly diminished by employing special drug delivery systems targeted specifically to cancer cells (Minko et al., 2004). Targeting is especially important in circumstances where a localized tumor is removed surgically, and chemotherapy is prescribed as a follow-up preventive against potential metastases (Dharap et al., 2005). Among the vehicles that can be used for special anticancer drug delivery are lipoproteins.

Lipoproteins have been considered appropriate drug-delivery vehicles for anticancer drugs (Braschi et al., 1999), owing to their structural features, biocompatibility and targeting capability via receptor mediated mechanisms (Nikanjam et al., 2007). The basic structure of lipoproteins, which comprises of an outer protein-phospholipid shell with a lipophilic surface and an interior hydrophobic compartment, positions them as ideal transporters of hydrophobic drugs, including anticancer agents. Due to their biocompatible, lipoproteins have considerable advantages over the conventional carrier systems currently used in cancer chemotherapy in that they provide the opportunity for targeted delivery of the anticancer drug they carry through endocytosis by receptor mediated uptake or by selective uptake of core components (Pathania, et al., 2003).

The main advantages of lipoprotein-based formulations are their biocompatible components, their relative stability in the blood circulation and their track record of having already been safely injected into human subjects (Bisoendial et al., 2002). In addition, 
lipoprotein-based formulations have vast targeting potential via receptor-mediated mechanisms that are overexpressed in cancer cells compared with normal cells. It has been shown that anticancer drugs can be targeted to cancer cells that generally express a high level of lipoprotein receptors, by encapsulation into reconstituted high density lipoprotein nanoparticles which are similar to the endogenous lipoprotein particles responsible for shuttling of hydrophobic molecules to different parts of the body.

Lipoprotein complexes are ideal for loading and targeted delivery of cancer therapeutic and diagnostic agents because they can mimic the shape and structure of endogenous lipoproteins, and as such, remain in circulation for an extended period of time, while largely evading the reticuloendothelial cells in the body's defenses. The small size (less than $30 \mathrm{~nm}$ ) of the low-density and high-density classes of lipoproteins allows them to maneuver deeply into tumors. Furthermore, lipoproteins can be targeted to their endogenous receptors, especially, when the receptors are implicated in cancer. Although the lipophilic character of certain pharmaceuticals may be a disadvantage during intravenous therapy, this can be advantageous in anticancer drug deliveries as the highly lipid compounds are ideally suited for incorporation into lipoproteins. Most of the 'orphaned' anticancer drugs that have failed primarily due to their poor water solubility can also be made to progress faster through development process by incorporating them into lipoproteins.

\subsubsection{Low density lipoprotein in cancer chemotherapy}

Low density lipoprotein (LDL) has been found to represent a suitable carrier for cytotoxic drugs that may target them to cancer (Kader and Pater, 2002). This is because the low-density lipoprotein receptor (LDLR) has been found to be over-expressed in numerous cancers. The upregulated levels of low-density receptor in these cancers are believed to provide the cancer cells with the necessary lipid substrates needed for active membrane synthesis. In fact, sequestration of plasma LDL cholesterol in cancers has been suggested to explain the low levels of circulating total and LDL cholesterol observed in patients with malignancies. These findings have led many researchers to investigate the possibility of exploiting LDL as a delivery vehicle for cancer diagnostics and therapeutics (Corbin and Zheng, 2007).

The high requirement of LDL by cancer cells and thus the overexpression of LDL receptor can be utilized for developing a novel targeted drug delivery system. This can be achieved by targeting of the LDL particle and allowing the anticancer drugs to be transferred to the natural LDL inside of the body. LDL will function as a secondary carrier of anticancer molecules and deliver these molecules selectively to cancerous cells via elevated LDL receptors. This approach requires the anticancer molecules to have affinity for the LDL particle endogenously and to have certain special physicochemical properties.

There are at least three different ways in which diagnostic or anticancer agents can be incorporated into LDL. The first of these is protein labeling in which the anticancer drug is covalently attached to the amino acid residues of apolipoprotein (apo) B-100 protein of LDL; the second is surface labeling which involves intercalation of the diagnostic or 
anticancer agent into the phospholipid monolayer of LDL; the third is reconstitution core loading via substitution of anticancer agents into the lipid core of LDL. A large number of researchers have shown that various anticancer agents could be actively incorporated into LDL through intercalation or reconstitution methods. Moreover, these novel LDL-drug complexes were shown to be more efficacious against cancer cells than their conventional counterparts.

Coupling of doxorubicin to human LDL to form a LDL-doxorubicin complex injected to mice resulted in greater accumulation of LDL-doxorubicin, in the liver, than free doxorubicin. In contrast, LDL- doxorubicin was less accumulated in heart than free doxorubicin. This suggests LDL could be used as carriers to conjugate anti-cancer drugs.

\subsubsection{Problems limiting the use of LDL as delivery vehicle}

Although a lot of studies have produced promising results, progress towards utilizing LDL as a delivery vehicle in the clinical setting has been impeded by the need to isolate LDL from fresh donor plasma. Relying on donor plasma to acquire LDL is problematic because LDL samples vary from batch to batch, methods for isolating LDL are lengthy and large quantities of LDL are difficult to attain. Furthermore, isolated LDL can only be stored for finite periods before aggregation and degradation processes compromise the integrity of the LDL sample. As a result of these limitations, attempts have been made to prepare synthetic LDL-like particles consisting of phospholipid/cholesterol ester microemulsions and apoB100 (the LDLR-binding component of LDL). Difficulties also plagued this endeavor owing to the size and complexity of the apoB-100 protein which is one of the largest monomeric proteins known consisting of over 4500 amino acids with a molecular weight of $550 \mathrm{kDa}$. Furthermore, apoB-100 is highly insoluble in aqueous solutions, making it difficult to work with. In addition, these problems are compounded still by the difficulties of having to isolate apoB-100 from donor plasma and other approaches to working with apoB-100 are therefore needed.

\subsubsection{High-density Lipoprotein in cancer chemotherapy}

The targeted delivery of anticancer agents via lipoprotein carriers is based on the concept that cancer cells have a higher expression of lipoprotein receptors (Lacko et al., 2002; Cao et al., 2004) due to their increased need for cholesterol to promote rapid proliferation. Clinical studies have shown that HDL cholesterol levels, like LDL levels, are lower in cancer patients, including those with haematological malignancies (Fiorenza et al., 2000). Unfortunately, the targeting of chemotherapeutic agents via HDL is daunting because overwhelming efforts are required for the isolation HDL from human plasma and a lot of biosafety concerns are attached with the injection of human-blood-derived products (Adams et al., 2003). Consequently, the focus of future studies is likely to be on synthetic/reconstituted lipoproteins with favourable drug-carrying capacity, and the exploitation of their potential for targeting tumour cells and tissues. 
HDL transports cholesterol to liver cells, where they are recognized and taken up via specific receptors. Cholesteryl esters within HDL are selectively uptaken by hepatocytes via the scavenger receptor class B type I (SR-BI). An interesting feature of SR-BI is that the receptor selectively translocates HDL-cholesteryl esters from the lipoprotein particle to the cytosol of the liver parenchymal cells without a parallel uptake of the apolipoproteins and this property may allow for the delivery of its loaded drugs avoiding lysosomal degradation (Lou et al., 2005). The high affinity of cancer cells for HDL has made them useful as carriers for delivering anticancer drugs into hepatoma cells to treat HCC. Anti-cancer drug-HDL complexes work as efficient drug delivery vehicles due to the ability of cancer cells to acquire HDL core components (Wasan et al., 1996). Complexing of anti-cancer drugs with HDL does not influence characteristics of the anticancer drugs (Kader et al., 2002) and administration of anti-cancer drug-HDL complex may reduce toxic side-effects during the chemotherapy( Lacko, et al.,2002).

In a cell culture system, cellular uptake of recombinant HDL-aclacinomycin by the SMMC-7721 hepatoma cells was significantly higher than that of free aclacinomycin at the concentration range of $0.5-10 \mu \mathrm{g} / \mathrm{mL}$. Cytotoxicity of recombinant HDL- aclacinomycin to the hepatoma cells was significantly higher than that of free aclacinomycin at concentration range of less than $5 \mu \mathrm{g} / \mathrm{mL}$ just as IC50 of recombinant HDL-aclacinomycin was lower than IC50 of free aclacinomycin (Lou et al., 2005). These results strongly suggest that HDL could be used as carriers to conjugate water-insoluble anti-cancer drugs in order to achieve higher therapeutic concentrations of the drugs in the microenvironment of the cancer cells.

\section{Conclusion}

Lipoproteins are complex endogenous aggregates of lipids and proteins that function primarily for the transport of water insoluble lipids from their point of origin to their respective destinations. Lipoproteins are classified as chylomicrons, very low density lipoproteins, low density lipoproteins and high density lipoproteins, based on the relative densities of the aggregates on ultracentrifugation.

Lipoproteins, which are cholesterol-rich particles, have been especially found to play significant roles in the pathogenesis of a large number of cancers because rapidly dividing cancerous cells generally have high requirements for cholesterol. This is exemplified by the increased LDL-requirement associated with a number of cancers including cancers of the prostate, colon, adrenal gland etc.

The efficacy of cancer chemotherapy is often limited by severe deleterious effects induced by anticancer drugs, on healthy and cancerous cells because of lack of specificity for the cancerous cells. In addition most available anticancer drugs do not perform optimally because of limited accessibility to target tissues. The deleterious effects of anticancer drugs on healthy organs can be markedly diminished by employing special drug delivery systems that specifically target cancer cells, using lipoproteins as carriers. 
The main advantages of lipoproteins as anti-cancer drug carriers are: (1) lipoproteins are spherical particles consisting of a core of apolar lipids surrounded by a phospholipid monolayer, in which cholesterol and apoproteins are embedded. Therefore, highly lipophilic drugs can be incorporated into the apolar core without affecting lipoprotein receptor recognition; (2) lipoproteins are completely bio-degradable, do not trigger immunological responses, escape from recognition and elimination by the reticuloendothelial system, and have a relatively long half-life in the circulation; (3) lipoproteins can be recognized and taken up via specific receptors, and can mediate cellular uptake of the carried drugs ; and (4) many cancer cells show a high ability of lipoprotein uptake and therefore high therapeutic levels of the conjugated drugs can be rapidly attained at the target site(s).

\section{Author details}

Adebowale Bernard Saba and Temitayo Ajibade

Department of Veterinary Physiology,

Biochemistry and Pharmacology, University of Ibadan, Ibadan, Nigeria

\section{References}

Adams, K. M.; Lamber, N.C.; Heimfeld S.; Tylee, T. S. \& Pang J. M. (2003). Male DNA in female donor apheresis and CD34-enriched products. Blood 102: 3845-3847. doi:10.1182/blood-2003-05-1570.

Ahaneku, J.E.; Taylor, G.O.; Olubuyide, I.O. \& Agbedana, E.O. (1992). Abnormal lipid and lipoprotein patterns in liver cirrhosis with and without hepatocellular carcinoma. J Pak Med Assoc, 42(11):260-263. PMID:1336073

Albers, J. J.; Tollefson J. H.;Chen, C. H. \& Steinmetz A. (1984) "Isolation and characterization of human plasma lipid transfer proteins," Arteriosclerosis; 4 (1): 49-58. PMID:6691846

Amemiya, H.; Arinami, T.; Kikuchi S.; Yamakawa-Kobayashi K.; Li L.; Fujiwara H. (1996). Apolipoprotein(a) and pentanucleotide repeat polymorphisms are associated with the degree of atherosclerosis in coronary heart disease. Atherosclerosis. 123:181-191. PMID:8782849

Barter, P.J.; Kastelein, \& Kastelein J. (2003). "High density lipoproteins (HDLs) and atherosclerosis; the unanswered questions," Atherosclerosis. 168 (2) 195-211. PMID:12801602

Bisoendial, R.J.; Hovingh, G.K \& de Groot, E. (2002). Measurement of subclinical atherosclerosis: beyond risk factor assessment. Curr Opin Lipidol 13:595-603. PMID:12441883

Blackman, J.D.; Cabana, V.G. \& Mazzone, T. (1993). The acute-phase response and associated lipoprotein abnormalities accompanying lymphoma. J Intern Med. 233(2):2014. PMID: 8433082

Bowden, J.F.; Pritchard, P.H.; Hill, J.S. \& Frohlich, J.J. (1994). Lp(a) concentration and apo(a) isoform size. Relation to the presence of coronary artery disease in familial hypercholesterolemia. Arterioscler Thromb 14:1561-1568. PMID:7918305 
Braschi, S.; Neville, T.A.; Vohl, M.C.\& Sparks, D.L. (1999). Apolipoprotein A-I charge and conformation regulate the clearance of reconstituted high density lipoprotein in vivo. J Lipid Res.4 0(3):522-532. PMID:10064741

Brown, W.V. (2007). High-density lipoprotein and transport of cholesterol and triglyceride in blood. J. Clin. Lipidology, 1, 7-19. PMID: 21291664

Byrne, C.D. \& Lawn, R.M. (1994). Studies on the structure and function of the apolipoprotein(a) gene. Clin Genet; 46:34-41. PMID:7988075

Cao, W.M.; Murao, K. \& Imachi H. (2004). A mutant high-density lipoprotein receptor inhibits proliferation of human breast cancer cells. Cancer Res; 64: 1515-1521. PMID:14973113

Chen, Y. \& Hughes-Fulford M. (2001). Human prostate cancer cells lack feedback regulation of low-density lipoprotein receptor and its regulator, SREBP2. Int J Cancer. 2001;91:4145. PMID:11149418

Cooper, M.E., Akdeniz A. \& Hardy K.J. (1996): Effects of liver transplantation and resection on lipid parameters: a longitudinal study. Aust N Z J Surg; 66(11):743-746. PMID:8918381

Corbin, I.R. \& Zheng G. (2007). Mimicking Nature's Nanocarrier: Synthetic Low-density Lipoprotein-like Nanoparticles for Cancer-drug Delivery. Nanomedicine ;2(3):375380. PMID:17716181

Dharap, S.S.; Wang, Y.; Chandna, P.; Khandare, J.J.; Qiu, B; Gunaseelan, S.; Sinko, P.J.; Stein, S; Farmanfarmaian, A. \& Minko, T. (2005). Tumor-specific targeting of an anticancer drug delivery system by LHRH peptide. PNAS 10236 12962-12967. PMID:16123131

Dullaart, R.P.F. \& van Tol, A. (2001). Role of phospholipid transfer protein and pre $\beta$-high density lipoproteins in maintaining cholesterol efflux from Fu5AH cells to plasma from insulin-resistant subjects. Scand. J. Clin. Lab. Invest.;61:69-74. PMID:11300613

Fiorenza, A.M.; Branchi, A. \& Sommariva, D. (2000). Serum lipoprotein profile in patients with cancer. A comparison with non-cancer subjects. Int J Clin Lab Res; 30:141-145. PMID:11196072

Furberg, A.; Marit, B.V.; Wilsgaard, T.; Bernstein, L.\& Thune, .I (2004). Serum High-Density Lipoprotein Cholesterol, Metabolic Profile, and Breast Cancer Risk. JNCI J Natl Cancer Inst 96 (15): 1152-1160. PMID: 15292387

Geiss, H.C.; Ritter, M.M.; Richter, W.O.; Schwandt, P.\& Zachoval, R. (1996). Low lipoprotein (a) levels during acute viral hepatitis. Hepatology.;24(6):1334-1337. PMID:8938156

Genest, J.J.; McNamara, J.R.; Ordovas, J.M.; Martin-Munley, S.; Jenner, J.L.; Millar, J.;Salem, D.N. \& Schaefer, E.J. (1990): Effect of elective hospitalization on plasma lipoprotein cholesterol and apolipoproteins A-I, B and Lp(a). Am J Cardiol, 65(9):677-679. PMID:2106773

Giuseppe, L.; Massimo F.; Gian L.S. \& Gian CG (2007). Lipoprotein[a] and cancer: Antineoplastic effect besides its cardiovascular potency. Cancer Treatment Reviews 33, 427436. doi:10.1016/j.ctrv.2007.02.006

Gotto, A.M. (2001). "Low high-density lipoprotein cholesterol as a risk factor in coronary heart disease: a working group report," Circulation; 103 (17) 2213-2218. PMID:11331265 
Hasstedt, S.J.; Ash, K.O. \& Williams R.R. (1986). A re-examination of major locus hypotheses for high density lipoprotein cholesterol level using 2,170 persons screened in 55 Utah pedigrees. Am J Med Genet;24:57-67. PMID:3706413

Hoyer, A. P.; \& Engholm G. (1992). "Serum lipids and breast cancer risk: a cohort study of 5,207 Danish women," Cancer Causes and Control; 3(5) 403-408. PMID:1525320

Jafri, H.; Alsheikh-Ali, A.A. \& Karas, R.H. (2010). Baseline and on-treatment high-density lipoprotein cholesterol and the risk of cancer in randomized controlled trials of lipidaltering therapy. J Am Coll Cardiol; 55:2846-54. PMID:21173414

Kader, A. \& Pater, A. (2002). Loading anticancer drugs into HDL as well as LDL has little affect on properties of complexes and enhances cytotoxicity to human carcinoma cells. $J$ Control Release; 80(1-3):29-44. PMID:11943385

Kanel, G.C.; Radvan, G. \& Peters RL (1983). High-density lipoprotein cholesterol and liver disease. Hepatology; 3: 343-348. PMID:6840679

Krempler. F.; Kostner, G.M.; Roscher, A.; Haslauer, F.;Bolzano, K. \&Sandhofer, F. (1983). Studies on the role of specific cell surface receptors in the removal of lipoprotein (a) in man. J Clin Invest.;71:1431-1441. PMID: 6304146

Kwiterovich, P.O. (2000). "The metabolic pathways of high-density lipoprotein, low-density lipoprotein, and triglycerides: a current review". The American journal of cardiology 86 (12A): 5L-10L. PMID: 11374859

Lacko, A.G.; Nair, M.; Paranjape, S.; Johnson, S. \& McConathy, W.J. (2002). High density lipoprotein complexes as delivery vehicles for anticancer drugs. Anticancer Res. 22(4):2045-9. PMID:12174882

Lacko, A.G.; Nair, M.; Prokai, L.\& McConathy, W.J. (2007).Prospects and challenges of the development of lipoprotein-based formulations for anti-cancer drugs. Expert Opin Drug Deliv.;4(6):665-75. PMID:17970668

Langstein, H.N. \& Norton, J.A. (1991). Mechanisms of cancer cachexia. Hematol Oncol Clin North Am. 5:103-123. PMID: 2026566

Li, W.X. (1993). [Serum cholesterol and cancer mortality: eleven-year prospective cohort study on more han nine thousand persons]. Zhonghua Liu Xing Bing Xue Za Zhi. 14(1):69. PMID:8504456

Lou, B.; Liao, X.L.; Wu, M.P.; Cheng, P.F.; Yin, C.Y. \& Fei, Z. (2005). High-density lipoprotein as a potential carrier for delivery of a lipophilic antitumoral drug into hepatoma cells. World J Gastroenterol; 11(7):954-959. PMID:15742395

Malaguarnera, M.; Trovato, G.; Restuccia, S.; Giugno, I., Franze, C.M., Receputo, G., Siciliano, R.; Motta, M. \& Trovato, B.A. (1994). Treatment of nonresectable hepatocellular carcinoma: review of the literature and meta-analysis. Adv Ther; 11:303319. PMID:10150270

Malaguarnera, M.; Giugno, I.; Trovato, B.A.; Panebianco, M.P.; Restuccia, N. \& Ruello, P. (1996). Lipoprotein(a) in cirrhosis. A new index of liver functions? Curr Med Res Opin; 13:479-485. PMID:8840366

Marcovina, S.M.; Zhang, Z.H.; Gaur, V.P. \& Albers, J.J. (1993). Identification of 34 apolipoprotein(a) isoforms: differential expression of apolipoprotein(a) alleles between American blacks and whites. Biochem Biophys Res Commun;191:1192-1196. PMID:8466495 
McLean, J.W.; Tomlinson, J.E.; Kuang, W.J.; Eaton, D.L.; Chen, E.Y. \& Fless, G.M. (1987). cDNA sequence of human apolipoprotein(a) is homologous to plasminogen. Nature;330:132-137. PMID:3670400

Mendez, A.J.; Oram, J.F.\& Bierman, E.L. (1991). Protein kinase C as a mediator of high density lipoprotein receptor-dependent efflux of intracellular cholesterol. J Biol Chem; 266. PMID:1645339

Minko, T.; Dharap, S.S.; Pakunlu, R.I.\& Wang, Y. (2004). Molecular targeting of drug delivery systems to cancer. Curr. Drug Targets; 5 389-406. PMID: 15134222

Moorman, P.G.; Hulka, B.S.; Hiatt, R.A.; Krieger, N.; Newman, B.; Vogelman, J.H. \& Orentreich, N. (1998). Association between high-density lipoprotein cholesterol and breast cancer varies by menopausal status. Cancer Epidemiol Biomarkers Prev;7(6):483-8. PMID: 9641492

Motta, M.; Giugno, I.; Ruello, P.; Pistone, G.; Di Fazio, I. \& Malaguarnera, M. (2001). Lipoprotein(a) behaviour in patients with hepatocellular carcinoma. Minerva Med.;92:301-305. PMID:11675573

Niendorf, A.; Nagele, H.; Gerding, D.; Meyer-Pannwitt, U. \& Gebhardt, A (1995) Increased LDL receptor mRNA expression in colon cancer is correlated with a rise in plasma cholesterol levels after curative surgery. Int J Cancer, 61: 461-464. PMID:7759150

Ng, C.J.; Wadleigh, D.J.; Gangopadhyay, A.; Hama, S.; Grijalva, V.R. \& Navab, M. (2001). Paraoxonase-2 is a ubiquitously expressed protein with antioxidant properties and is capable of preventing cell-mediated oxidative modification of low density lipoprotein. $J$ Biol Chem ; 276:44444-9. PMID:11579088.

Nikanjam, M.; Gibbs, A.R.; Hunt, C.A.; Budinger, T.F. \& Forte, T.M. (2007). Synthetic nanoLDL with paclitaxel oleate as a targeted drug delivery vehicle for glioblastoma multiforme. J Control Release, 124:163-171. PMID:17964677

Pathania, D.; Millard, M. \& Neamati N (2009). Opportunities in discovery and delivery of anticancer drugs targeting mitochondria and cancer cell metabolism. Adv Drug Deliv.,30;61(14):1250-75. PMID:19716393

Perletti, G.; Tessitore, L.; Sesca, E.; Pani, P.; Dianzani, M.U. \& Piccinini, F. (1996). Epsilon PKC acts like a marker of progressive malignancy in rat liver, but fails to enhance tumorigenesis in rat hepatoma cells in culture. Biochem Biophys Res Commun; 221: 688691. PMID: 8630022

Perri, S.R.; Martineau, D.; François, M.; Bisson, L.; Durocher, Y. \& Galipeau, J. (2007). Plasminogen Kringle 5 blocks tumor progression by antiangiogenic and proinflammatory pathways. Mol Cancer Ther;6 (2):441-9. doi:10.1158/1535-7163.MCT-060434

Reddy, S.T.; Wadleigh, D.J.; Grijalva, V., Ng, C., Hama, S. \& Gangopadhyay A (2001). Human paraoxonase-3 is an HDL-associated enzyme with biological activity similar to paraoxonase-1 protein but is not regulated by oxidized lipids. Arterioscler Thromb Vasc Biol;21:542-7. PMID:11304470

Romics, L.; Nemesánszky, E.; Szalay, F.; Császár, A.; Tresch, J. \& Karádi, I. (1996). Lipoprotein(a) concentration and phenotypes in primary biliary cirrhosis. Clin Chim Acta;255:165-171. PMID:8937759 
Rosen, H. \& Abribat T (2005). The rise and rise of drug delivery. Nat Rev Drug Discov. ;4:381-5. PMID: 15864267

Rudling, M.J.; Angelin, B.; Peterson, C.O. \& Collins, V.P. (1990). Low density lipoprotein receptor activity in human intracranial tumors and its relation to the cholesterol requirement. Cancer Res. 50, 483-487. Available from http://cancerres.aacrjournals.org/content/50/3/483\#related-urls

Samonakis, D.N.; Koutroubaki, I.E.; Sfiridaki, A; Malliaraki, N.; Antoniou, P.\& Romanos J (2004). Hypercoagulable states in patients with hepatocellular carcinoma. Dig Dis Sci; 49:854-858. PMID: 15259509

Sehouli, J.; Stengel, D.; Elling, D.; Ortmann, O.; Blohmer, J. Riess, H. \&Lichtenegger W (2002) Gynecol. Oncol., 85 , 321-326. PMID: 11972395

Shah, P.K.; Kaul, S.; Nilsson, J. \& Cercek, B. (2001). “Exploiting the vascular protective effects of high-density lipoprotein and its apolipoproteins: an idea whose time for testing is coming, part I," Circulation, 104 (19). 2376-2383. PMID:11696481

Skinner, E. R. (1994). "High-density lipoprotein subclasses," Current Opinion in Lipidology, 5 (3). 241-247. doi:10.1155/2011/496925

Spiegel, D.; Bloom, J.R.; Kramer, H.C. \& Gottheil, E. (1989). Effect of treatment on the survival of patients with metastasic breast cancer. Lancet, 2, 888-891. PMID: 9885092

Uccello, M.; Malaguarnera, G.; Pelligra, E.M.; Biondi, A.; Basile, F. \& Motta M (2011). Lipoprotein(a) as a potential marker of residual liver function in hepatocellular carcinoma. Indian J Med Paediatr Oncol.; 32(2): 71-75. PMID:22174493

Utermann, G; Duba, C.\& Menzel, H.J. (1988). Genetics of the quantitative Lp(a) lipoprotein trait. II. Inheritance of Lp(a) glycoprotein phenotypes. Hum Genet;78:47-50. PMCID: PMC287411

Vitols, S.; Peterson, C.; Larsson, 0; Holm, P. \& Aberg B (1992). Elevated uptake of low density lipoproteins by human lung cancer tissue in vivo. Cancer Res., 52, 6244-6247. 0007-0920/95

Wade, D.P.; Clarke, J.G.; Lindahl, G.E.; Liu, A.C.; Zysow, B.R.\& Meer, K. (1993) 1. 5' control regions of the apolipoprotein(a) gene and members of the related plasminogen gene family. Proc Natl Acad Sci U S A;90:1369-1373. PMID:7679504

Wasan, K.M. \& Morton RE (1996): Differences in lipoprotein concentration and composition modify the plasma distribution of free and liposomal annamycin. Pharm Res., 13(3):462468. DOI: $10.1021 /$ js $960495 j$

Yokoyama, S. (2005). Assembly of high density lipoprotein by the ABCA1/apolipoprotein pathway. Curr. Opin. Lipidol.;16:269-279. Downloaded from www.jlr.org 